LA-UR-01-6876

Approved for public release; distribution is unlimited.

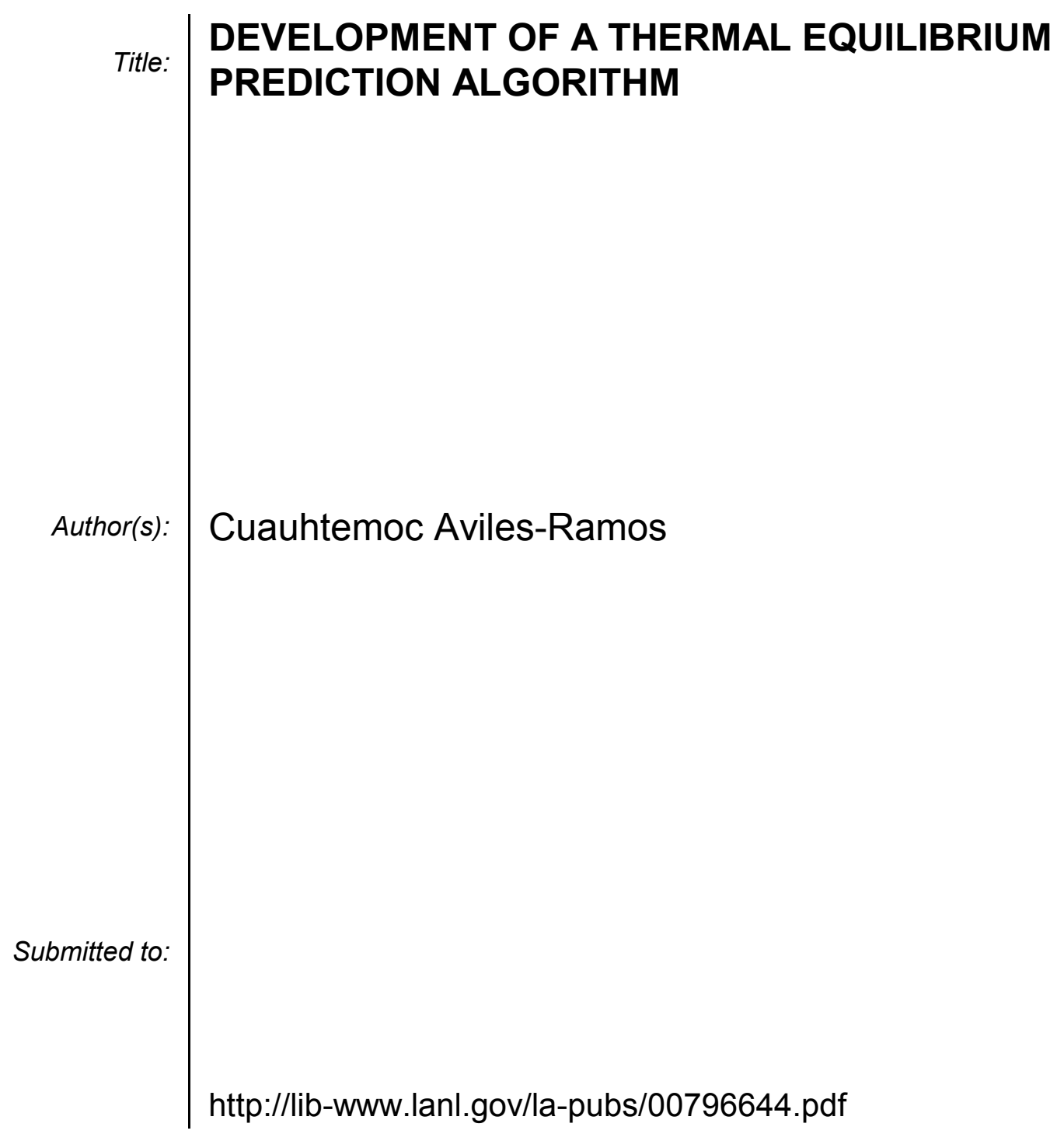

Los Alamos National Laboratory, an affirmative action/equal opportunity employer, is operated by the University of California for the U.S. Department of Energy under contract W-7405-ENG-36. By acceptance of this article, the publisher recognizes that the U.S. Government retains a nonexclusive, royaltyfree license to publish or reproduce the published form of this contribution, or to allow others to do so, for U.S. Government purposes. Los Alamos National Laboratory requests that the publisher identify this article as work performed under the auspices of the U.S. Department of Energy. Los Alamos National Laboratory strongly supports academic freedom and a researcher's right to publish; as an institution, however, the Laboratory does not endorse the viewpoint of a publication or guarantee its technical correctness. 


\title{
DEVELOPMENT OF A THERMAL EQUILIBRIUM PREDICTION ALGORITHM
}

\author{
Cuauhtemoc Aviles-Ramos \\ Safeguards Science and \\ Technology, NIS-5 \\ Los Alamos National Laboratory
}

\begin{abstract}
A thermal equilibrium prediction algorithm is developed and tested using a heat conduction model and data sets from calorimetric measurements. The physical model used in this study is the exact solution of a system of two partial differential equations that govern the heat conduction in the calorimeter. A multi-parameter estimation technique is developed and implemented to estimate the effective volumetric heat generation and thermal diffusivity in the calorimeter measurement chamber, and the effective thermal diffusivity of the heat flux sensor. These effective properties and the exact solution are used to predict the heat flux sensor voltage readings at thermal equilibrium. Thermal equilibrium predictions are carried out considering only $20 \%$ of the total measurement time required for thermal equilibrium. A comparison of the predicted and experimental thermal equilibrium voltages shows that the average percentage error from 330 data sets is only $0.1 \%$. The data sets used in this study come from calorimeters of different sizes that use different kinds of heat flux sensors. Furthermore, different nuclear material matrices were assayed in the process of generating these data sets. This study shows that the integration of this algorithm into the calorimeter data acquisition software will result in an $80 \%$ reduction of measurement time. This reduction results in a significant cutback in operational costs for the calorimetric assay of nuclear materials.
\end{abstract}

\section{INTRODUCTION}

A simple physical model for a calorimeter was developed by C. Aviles-Ramos [1]. A parameter estimation problem is solved using this model and the voltage signal from the calorimeter heat flux sensor. This model assumes that the calorimeter is divided into two cylindrical regions and that heat is transferred by conduction. However, the estimated thermophysical properties also contain the effects of convection and radiation heat transfer that could be present in the calorimeter. A hybrid algorithm developed by C. Aviles-Ramos [2] is used to solve a parameter estimation problem that involves the calculation of the effective thermophysical properties of the two cylindrical regions. The hybrid algorithm takes advantage of the linearity of the heat conduction model. This algorithm separates the linear and nonlinear dependence of the heat conduction model on the parameters to be estimated. It consists of a linear parameter estimation solver and a nonlinear minimization algorithm. These algorithms are put into communication through a FORTRAN function subprogram. This programming structure allows different nonlinear minimization algorithms to be tested while keeping the same linear parameter estimation solver.

\section{NOMENCLATURE}

$a$ inner radius of power sensor, $\mathrm{cm}$

$b$ outer radius of power sensor, $\mathrm{cm}$

$c_{p 1}$ heat capacity of region $1, \mathrm{~J} /(\mathrm{kg} \mathrm{K})$

$c_{p 2}$ heat capacity of region $2, \mathrm{~J} /(\mathrm{kg} \mathrm{K})$

$d$ height of power sensor, $\mathrm{cm}$

$e \quad$ percentage error defined by Eq. (43)

$C_{m 0}$ coefficients, Eqs. (16) or (18)

$D_{m 0}$ coefficients, Eq. (17) or (19)

$C_{1} \quad$ symbol that represents $g_{0}$

$C_{2} \quad$ symbol that represents $\alpha_{3}$

$C_{3} \quad$ symbol that represents $\alpha_{4}$

$F_{1, m n}$ eigenfunction in region 1

$F_{2, m n}$ eigenfunction in region 2

$F_{1, m n}^{*}$ eigenfunction in region 1 for $\alpha_{1}<\alpha_{z 2}$

$F_{2, m n}^{*}$ eigenfunction in region 2 for $\alpha_{1}<\alpha_{z 2}$ 
$g_{0} \quad$ volumetric heat generation, $\mathrm{W} / \mathrm{cm}^{3}$

$J_{0} \quad$ Bessel function of the first kind and order zero

$k_{r 2}$ thermal conductivity in region 2 along $r$-direction, $\mathrm{W} /(\mathrm{cm} \mathrm{K})$

$k_{z 2}$ thermal conductivity in region 2 along $z$-direction, $\mathrm{W} /(\mathrm{cm} \mathrm{K})$

$k_{1}$ thermal conductivity in region $1, \mathrm{~W} /(\mathrm{cm} \mathrm{K})$

$N_{m 0}$ norm, Eq. (21)

$N_{m 0}^{*}$ modified norm, Eq. (23)

$N_{m n}^{*}$ norm calculated using $F_{1, m n}^{*}$ and $F_{2, m n}^{*}$

$P \quad$ predicted thermal equilibrium, Watts or Volts

$q_{z 1}$ heat flux at $z=0$ in $0<r<a, \mathrm{~W} / \mathrm{cm}^{2}$

$q_{z 2}$ heat flux at $z=d$ in $0<r<a, \mathrm{~W} / \mathrm{cm}^{2}$

$t$ time, s

$t_{f} \quad$ final prediction time, $\mathrm{s}$

$T_{2}$ temperature in power sensor, ${ }^{\circ} \mathrm{C}$

$T_{s} \quad$ prescribed surface temperature, ${ }^{\circ} \mathrm{C}$

$T_{0, i} \quad$ initial temperature in regions 1 or $2,{ }^{\circ} \mathrm{C}$

$r$ coordinate, $\mathrm{cm}$

$Y_{0} \quad$ Bessel function of the second kind and order zero

$z \quad$ coordinate, $\mathrm{cm}$

\section{Greek}

$\alpha_{z 2}$ thermal diffusivity in region 2 , in $z$-direction, $\mathrm{cm}^{2} / \mathrm{s}$

$\alpha_{r 2}$ thermal diffusivity in region 2 , in $r$-direction, $\mathrm{cm}^{2} / \mathrm{s}$

$\alpha_{1}$ thermal diffusivity in region $1, \mathrm{~cm}^{2} / \mathrm{s}$

$\alpha_{3} \quad \rho_{1} c_{p 1}\left(T_{0,1}-T_{s}\right), \mathrm{J} / \mathrm{cm}^{3}$

$\alpha_{4} \quad \rho_{2} c_{p 2}\left(T_{0,2}-T_{s}\right), \mathrm{J} / \mathrm{cm}^{3}$

$\gamma_{m n}$ eigenvalue for the $r$-direction in region $2, \mathrm{~cm}^{-1}$

$\gamma_{m n}^{*} \quad$ eigenvalue for the $r$-direction in region 2 for $\alpha_{1}<\alpha_{z 2}$, $\mathrm{cm}^{-1}$

$\eta_{m n}$ eigenvalue for the $r$-direction in region $1, \mathrm{~cm}^{-1}$

$\eta_{m n}^{*} \quad$ eigenvalue for the $r$-direction in region 1 for $\alpha_{1}<\alpha_{z 2}$, $\mathrm{cm}^{-1}$

$\lambda_{m n}$ eigenvalue for time, $s^{-1 / 2}$

$\lambda_{m n}^{*}$ eigenvalue for time, $s^{-1 / 2}$

$\Theta$ heat flux sensor signal, Volts or Watts

$\xi(n)$ integer function

$\Psi_{1} \quad$ function defined by Eq. (26)
$\Psi_{2} \quad$ function defined by Eq. (27)

$\Psi_{3} \quad$ function defined by Eq. (28)

\section{HEAT CONDUCTION MODEL}

The geometry used to develop the calorimeter model is shown in Fig. 1. The heat conduction equation in the isotropic inner cylinder shown in Fig. 1 is

$$
\begin{gathered}
k_{1} \frac{1}{r} \frac{\partial}{\partial r}\left(r \frac{\partial T_{1}}{\partial r}\right)+k_{1} \frac{\partial^{2} T_{1}}{\partial z^{2}} \\
+g_{1}(r, z, t)=\rho_{1} c_{p 1} \frac{\partial T_{1}}{\partial t} \text { in } 0<r<a
\end{gathered}
$$

Also, the diffusion equation in the orthotropic outer layer has the form

$$
\begin{gathered}
k_{r 2} \frac{1}{r} \frac{\partial}{\partial r}\left(r \frac{\partial T_{2}}{\partial r}\right)+k_{z 2} \frac{\partial^{2} T_{2}}{\partial z^{2}} \\
+g_{2}(r, z, t)=\rho_{2} c_{p 2} \frac{\partial T_{2}}{\partial t} \text { in } a<r<b
\end{gathered}
$$

where $g_{1}(r, z, t)$ and $g_{2}(r, z, t)$ are volumetric heat source functions. The volumetric heat generation function $g_{2}(r, z, t)$ could include the heat flux at the surface $r=b$. Subscripts 1 and 2 indicate the inner and outer cylinders respectively. Equations (1) and (2) are subjected to the following boundary and initial conditions

$$
\begin{gathered}
\frac{\partial T_{1}}{\partial r}=0 \text { at } r=0 \\
k_{1} \frac{\partial T_{1}}{\partial z}=q_{z 1}(r, t) \text { at } z=0 \text { in } 0<r<a \\
-k_{1} \frac{\partial T_{1}}{\partial z}=q_{z 2}(r, t) \text { at in } 0<r<a \\
k_{z 2} \frac{\partial T_{2}}{\partial z}=0 \text { at } z=0 \text { and } z=d \text { in } a<r<b \\
T_{2}(b, z, t)=T_{s}(z, t) \text { in } 0<z<d \\
T_{1}(r, z, 0)=f_{1}(r, z) \text { and } T_{2}(r, z, 0)=f_{2}(r, z)
\end{gathered}
$$

The solution of the system represented by Eqs. (1) and (2) was found in reference [1] and it is used as the physical model for this thermal equilibrium prediction research. Since the voltage signal of the calorimeter power sensor is proportional to the heat flux at the inner or outer surface of the outer cylinder (see Fig. 1), the temperature solution, $T_{2}$, found in reference [1] is differentiated with respect to $r$ to obtain the heat flux at the surface as 


$$
\begin{aligned}
& -\left.k_{r 2} \frac{\partial T_{2}}{\partial r}\right|_{r=b}=-k_{r 2} g_{0} d \sum_{m=1}^{\infty} \frac{a J_{1}\left(\eta_{m 0} a\right)}{\eta_{m 0} \lambda_{m 0}^{2} N_{m 0}} \\
& \times\left.\frac{\partial F_{2, m 0}}{\partial r}\right|_{r=b}\left[1-\exp \left(-\lambda_{m 0}^{2} t\right)\right] \\
& -k_{r 2} \rho_{1} c_{p 1}\left(T_{0,1}-T_{s}\right) \sum_{m=1}^{\infty} \frac{\exp \left(-\lambda_{m 0}^{2} t\right)}{N_{m 0}} \frac{a d J_{1}\left(\eta_{m 0} a\right)}{\eta_{m 0}} \\
& \times\left.\frac{\partial F_{2, m 0}}{\partial r}\right|_{r=b} \\
& -\frac{\rho_{2} c_{p 2}\left(T_{0,2}-T_{s}\right) k_{r 2}^{3 / 2}}{\sqrt{k_{z 2}}} \sum_{m=1}^{\infty} \frac{d \exp \left(-\lambda_{m 0}^{2} t\right)}{\gamma_{m 0} N_{m 0}} \\
& \times\left.\frac{\partial F_{2, m 0}}{\partial r}\right|_{r=b}\left\{C_{m 0}\left[b J_{1}\left(\gamma_{m 0} \bar{b}\right)-a J_{1}\left(\gamma_{m 0} \bar{a}\right)\right]\right. \\
& \left.+D_{m 0}\left[b Y_{1}\left(\gamma_{m 0} \bar{b}\right)-a Y_{1}\left(\gamma_{m 0} \bar{a}\right)\right]\right\} \\
& +\left.k_{r 2} a \sum_{m=1}^{\infty} \sum_{n=0}^{\infty} \frac{J_{1}\left(\eta_{m n} a\right)}{\lambda_{m n}^{2} N_{m n} \eta_{m n}} \frac{\partial F_{2, m n}}{\partial r}\right|_{r=b} \\
& \times\left[q_{z 1}+q_{z 2} \cos (n \pi)\right]\left[1-\exp \left(-\lambda_{m n}^{2} t\right)\right] \\
& +\left.k_{r 2} a \sum_{m=1}^{\xi(n)} \sum_{n=1}^{\infty} \frac{J_{1}\left(\eta_{m n}^{*} a\right)}{\lambda_{m n}^{* 2} N_{m n}^{*} \eta_{m n}^{*}} \frac{\partial F_{2, m n}^{*}}{\partial r}\right|_{r=b} \\
& {\left[q_{z 1}+q_{z 2} \cos (n \pi)\right]\left[1-\exp \left(-\lambda_{m n}^{* 2} t\right)\right] \text { for } \alpha_{1}<\alpha_{z 2}(9)}
\end{aligned}
$$

The definitions of all the parameters appearing in Eq. (9) are given in [1]. The last two terms of Eq. (9) contain $q_{z 1}$ and $q_{z 2}$ which represent the heat losses through the top and bottom surfaces of the inner enclosure of the calorimeter. If we had a heat flux sensor that produced at least two voltage signals that depend in the $z$-direction, it would be possible to keep the last two terms of Eq. (9). For example, we could have a heat flux sensor manufactured in such a way that a voltage signal would correspond to the region $0<z<d / 2$ and another voltage signal that would correspond to the region $d / 2<z<d$. This would make the estimation of the parameters $q_{z 1}$ and $q_{z 2}$ possible. Since the heat flux sensors we have available produce only one voltage signal independent of the $z$-direction, the last

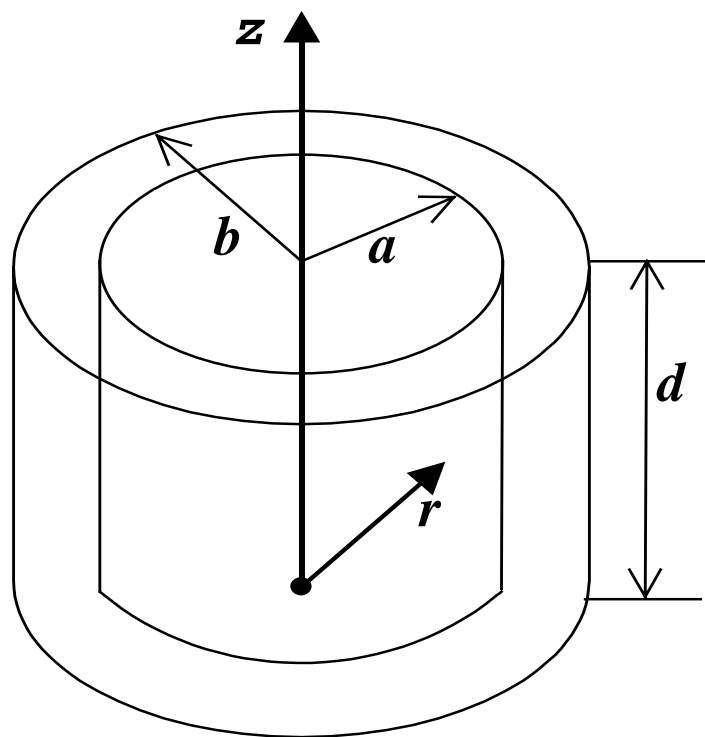

Figure 1. Two-domain calorimeter cylindrical geometry. The region defined by $\{0 \leq r \leq a$ and $0 \leq z \leq d\}$ represents the measurement chamber and the region located in $\{a \leq r \leq b$ and $0 \leq z \leq d\}$ represents the heat flux sensor.

two terms of Eq. (9) are neglected for the purposes of this study. Neglecting the last two terms of Eq. (9), the form of the model for the thermal equilibrium prediction is given by

$$
\begin{gathered}
-\left.k_{r 2} \frac{\partial T_{2}}{\partial r}\right|_{r=b}=-k_{r 2} g_{0} d \sum_{m=1}^{\infty} \frac{a J_{1}\left(\eta_{m 0} a\right)}{\eta_{m 0} \lambda_{m 0}^{2} N_{m 0}} \\
\times\left.\frac{\partial F_{2, m 0}}{\partial r}\right|_{r=b}\left[1-\exp \left(-\lambda_{m 0}^{2} t\right)\right] \\
-k_{r 2} \rho_{1} c_{p 1}\left(T_{0,1}-T_{s}\right) \sum_{m=1}^{\infty} \frac{\exp \left(-\lambda_{m 0}^{2} t\right)}{N_{m 0}} \frac{a d J_{1}\left(\eta_{m 0} a\right)}{\eta_{m 0}} \\
-\left.\left.\rho_{2} c_{p 2}\left(T_{0,2}-T_{s}\right) k_{r 2} \sum_{m=1}^{\infty} \frac{\partial F_{2, m 0}}{\partial r}\right|_{r=b}\right|_{m 0} N_{m 0} \\
\times\left.\frac{\partial F_{2, m 0}}{\partial r}\right|_{r=b}\left\{C_{m 0}\left[b J_{1}\left(\gamma_{m 0} \bar{b}\right)-a J_{1}\left(\gamma_{m 0} \bar{a}\right)\right]\right. \\
\left.+D_{m 0}\left[b Y_{1}\left(\gamma_{m 0} \bar{b}\right)-a Y_{1}\left(\gamma_{m 0} \bar{a}\right)\right]\right\}
\end{gathered}
$$


The heat flux given by Eq. (10) depends on $\rho_{1} c_{p 1}, \rho_{2} c_{p 2}$, $k_{1}, k_{r 2}, k_{z 2} T_{0,1}, T_{0,2}, T_{s}$, and $g_{0}$. This represents a total of 9 parameters. Since the objective is to predict thermal equilibrium and not to estimate actual thermophysical properties, Eq. (10) is modified to reduce the number of constants. A reduction of the number of parameters is possible because of the following identity

$$
\frac{k_{1}}{k_{r 2}} \frac{\sqrt{\alpha_{r 2}}}{\sqrt{\alpha_{1}}}=\frac{\rho_{1} c_{p 1}}{\rho_{2} c_{p 2}} \frac{\sqrt{\alpha_{1}}}{\sqrt{\alpha_{r 2}}}
$$

The eigenfunctions contained in the model equation (10) are defined as

$$
\begin{gathered}
F_{1, m 0}(r)=J_{0}\left(\eta_{m 0} r\right) \\
F_{2, m 0}(r)=\left[C_{m 0} J_{0}\left(\gamma_{m 0} r\right)+D_{m 0} Y_{0}\left(\gamma_{m 0} r\right)\right]
\end{gathered}
$$

where $\eta_{m 0}=\lambda_{m 0} / \sqrt{\alpha_{1}}, \quad \gamma_{m 0}=\lambda_{m 0} / \sqrt{\alpha_{r 2}}$, and $C_{m 0}$ and $D_{m 0}$ are given by

$$
\begin{gathered}
C_{m 0}=-\frac{\pi \gamma_{m 0} a}{2} J_{0}\left(\eta_{m 0} a\right) Y_{1}\left(\gamma_{m 0} a\right) \\
+\frac{\pi \eta_{m 0} k_{1} a}{2 k_{r 2}} J_{1}\left(\eta_{m 0} a\right) Y_{0}\left(\gamma_{m 0} a\right) \\
D_{m 0}=\frac{\pi \gamma_{m 0} a}{2} J_{0}\left(\eta_{m 0} a\right) J_{1}\left(\gamma_{m 0} a\right) \\
-\frac{\pi \eta_{m 0} k_{1} a}{2 k_{r 2}} J_{1}\left(\eta_{m 0} a\right) J_{0}\left(\gamma_{m 0} a\right)
\end{gathered}
$$

Using the definitions of $\eta_{m 0}$ and $\gamma_{m 0}$, Eqs. (14) and (15) can be written as

$$
\begin{array}{r}
C_{m 0}=\frac{1}{\sqrt{\alpha_{r 2}}}\left[-\frac{\pi \lambda_{m 0} a}{2} J_{0}\left(\eta_{m 0} a\right) Y_{1}\left(\gamma_{m 0} a\right)\right. \\
\left.+\frac{k_{1}}{k_{r 2}} \frac{\sqrt{\alpha_{r 2}}}{\sqrt{\alpha_{1}}} \frac{\pi a}{2} \lambda_{m 0} J_{1}\left(\eta_{m 0} a\right) Y_{0}\left(\gamma_{m 0} a\right)\right] \\
D_{m 0}=\frac{1}{\sqrt{\alpha_{r 2}}}\left[\frac{\pi \lambda_{m 0} a}{2} J_{0}\left(\eta_{m 0} a\right) J_{1}\left(\gamma_{m 0} a\right)\right. \\
\left.-\frac{k_{1}}{k_{r 2}} \frac{\sqrt{\alpha_{r 2}}}{\sqrt{\alpha_{1}}} \frac{\pi a}{2} \lambda_{m 0} J_{1}\left(\eta_{m 0} a\right) J_{0}\left(\gamma_{m 0} a\right)\right]
\end{array}
$$

The substitution of identity (11) into Eqs. (16) and (17) makes $C_{m 0}$ and $D_{m 0}$ functions of $\alpha_{1}, \alpha_{r 2}$, and $\rho_{1} c_{p 1} /\left(\rho_{2} c_{p 2}\right)$

$$
\begin{gathered}
C_{m 0}=\frac{1}{\sqrt{\alpha_{r 2}}}\left[-\frac{\pi \lambda_{m 0} a}{2} J_{0}\left(\eta_{m 0} a\right) Y_{1}\left(\gamma_{m 0} a\right)\right. \\
\left.+\frac{\rho_{1} c_{p 1}}{\rho_{2} c_{p 2}} \frac{\sqrt{\alpha_{1}}}{\sqrt{\alpha_{r 2}}} \frac{\pi a}{2} \lambda_{m 0} J_{1}\left(\eta_{m 0} a\right) Y_{0}\left(\gamma_{m 0} a\right)\right] \\
D_{m 0}=\frac{1}{\sqrt{\alpha_{r 2}}}\left[\frac{\pi \lambda_{m 0} a}{2} J_{0}\left(\eta_{m 0} a\right) J_{1}\left(\gamma_{m 0} a\right)\right. \\
\left.-\frac{\rho_{1} c_{p 1}}{\rho_{2} c_{p 2}} \frac{\sqrt{\alpha_{1}}}{\sqrt{\alpha_{r 2}}} \frac{\pi a}{2} \lambda_{m 0} J_{1}\left(\eta_{m 0} a\right) J_{0}\left(\gamma_{m 0} a\right)\right]
\end{gathered}
$$

From Eqs. (10) and (11), and the definitions of $\eta_{m 0}$ and $\gamma_{m 0}$, the eigenfunctions given by Eqs. (12) and (13) also become functions of $\alpha_{1}, \alpha_{r 2}$, and $\rho_{1} c_{p 1} /\left(\rho_{2} c_{p 2}\right)$. As a consequence of the definitions of $C_{m 0}$ and $D_{m 0}$ given by Eqs. (18) and (19), the transcendental equation (34) given in [1] becomes

$$
\begin{gathered}
\frac{\pi a \lambda_{m 0}}{2 \sqrt{\alpha_{r 2}}}\left\{J _ { 0 } ( \frac { \lambda _ { m 0 } a } { \sqrt { \alpha _ { 1 } } } ) \left[J_{1}\left(\frac{\lambda_{m 0} a}{\sqrt{\alpha_{r 2}}}\right) Y_{0}\left(\frac{\lambda_{m 0} b}{\sqrt{\alpha_{r 2}}}\right)\right.\right. \\
\left.-Y_{1}\left(\frac{\lambda_{m 0} a}{\sqrt{\alpha_{r 2}}}\right) J_{0}\left(\frac{\lambda_{m 0} b}{\sqrt{\alpha_{r 2}}}\right)\right]+\frac{\rho_{1} c_{p 1}}{\rho_{2} c_{p 2}} \frac{\sqrt{\alpha_{1}}}{\sqrt{\alpha_{r 2}}} \\
J_{1}\left(\frac{\lambda_{m 0} a}{\sqrt{\alpha_{1}}}\right)\left[Y_{0}\left(\frac{\lambda_{m 0} a}{\sqrt{\alpha_{r 2}}}\right) J_{0}\left(\frac{\lambda_{m 0} b}{\sqrt{\alpha_{r 2}}}\right)\right. \\
\left.\left.-J_{0}\left(\frac{\lambda_{m 0} a}{\sqrt{\alpha_{r 2}}}\right) Y_{0}\left(\frac{\lambda_{m 0} b}{\sqrt{\alpha_{r 2}}}\right)\right]\right\}=0
\end{gathered}
$$

It can be seen from transcendental equation (20) that the eigenvalues $\lambda_{m 0}$ also depend on $\alpha_{1}, \alpha_{r 2}$, and $\rho_{1} c_{p 1} /\left(\rho_{2} c_{p 2}\right)$. For $n=0$, the norm defined by Eq. (40) of reference [1] can be written as

$$
N_{m 0}=\int_{r=0}^{a} \int_{z=0}^{d} \rho_{1} c_{p 1}\left(F_{1, m 0}\right)^{2} r d r d z
$$




$$
+\int_{r=a}^{b} \int_{z=0}^{d} \rho_{2} c_{p 2}\left(F_{2, m 0}\right)^{2} r d r d z
$$

Because the definitions given by Eqs. (18) and (19) make $F_{1, m 0}$ and $F_{2, m 0}$ dependent on $\alpha_{1}, \alpha_{r 2}$, and $\rho_{1} c_{p 1} /\left(\rho_{2} c_{p 2}\right)$, the factorization of $\rho_{2} c_{p 2}$ in Eq. (21) produces a norm that is dependent on $\alpha_{1}, \alpha_{r 2}, \rho_{1} c_{p 1} /\left(\rho_{2} c_{p 2}\right)$, and $\rho_{2} c_{p 2}$

$$
N_{m 0}=\rho_{2} c_{p 2} N_{m 0}^{*}
$$

where $N_{m 0}^{*}$ is given by

$$
\begin{aligned}
N_{m 0}^{*} & =\frac{\rho_{1} c_{p 1}}{\rho_{2} c_{p 2}} \int_{r=0}^{a} \int_{z=0}^{d}\left(F_{1, m 0}\right)^{2} r d r d z \\
& +\int_{r=a}^{b} \int_{z=0}^{d}\left(F_{2, m 0}\right)^{2} r d r d z
\end{aligned}
$$

The substitution of Eqs. (12), (13), (18), (19), and (22) into Eq. (9) makes it possible to experess the heat flux at the surface of the sensor as a function of 6 parameters

$$
\begin{gathered}
-\left.k_{r 2} \frac{\partial T_{2}}{\partial r}\right|_{r=b}=-\alpha_{r 2} g_{0} d \sum_{m=1}^{\infty} \frac{a J_{1}\left(\eta_{m 0} a\right)}{\eta_{m 0} \lambda_{m 0}^{2} N_{m 0}^{*}} \\
\times\left.\frac{\partial F_{2, m 0}}{\partial r}\right|_{r=b}\left[1-\exp \left(-\lambda_{m 0}^{2} t\right)\right] \\
-\left.\alpha_{r 2} \alpha_{3} \sum_{m=1}^{\infty} \frac{\exp \left(-\lambda_{m 0}^{2} t\right)}{N_{m 0}^{*}} \frac{a d J_{1}\left(\eta_{m 0} a\right)}{\eta_{m 0}} \frac{\partial F_{2, m 0}}{\partial r}\right|_{r=b} \\
-\left.\alpha_{r 2} \alpha_{4} \sum_{m=1}^{\infty} \frac{d \exp \left(-\lambda_{m 0}^{2} t\right)}{\gamma_{m 0} N_{m 0}^{*}} \frac{\partial F_{2, m 0}}{\partial r}\right|_{r=b} \\
\quad \times\left\{C_{m 0}\left[b J_{1}\left(\gamma_{m 0} b\right)-a J_{1}\left(\gamma_{m 0} a\right)\right]\right. \\
\left.D_{m 0}\left[b Y_{1}\left(\gamma_{m 0} b\right)-a Y_{1}\left(\gamma_{m 0} a\right)\right]\right\}
\end{gathered}
$$

Thus, the heat flux at the surface of the sensor becomes a function of the form

$$
-\left.k_{r 2} \frac{\partial T_{2}}{\partial r}\right|_{r=b}=f\left(\alpha_{1}, \alpha_{r 2}, \frac{\rho_{1} c_{p 1}}{\rho_{2} c_{p 2}}, \alpha_{3}, \alpha_{4}, g_{0}\right)
$$

where $\alpha_{3}=\rho_{1} c_{p 1}\left(T_{0,1}-T_{s}\right)$ and $\alpha_{4}=\rho_{2} c_{p 2}\left(T_{0,2}-T_{s}\right)$. The values of $\rho_{1} c_{p 1}, \rho_{2} c_{p 2}, T_{0,1}, T_{0,2}$, and $T_{s}$ don't need to be determined individually because the objective here is to predict the onset of thermal equilibrium. These parameters are lumped into the ratio $\rho_{1} c_{p 1} /\left(\rho_{2} c_{p 2}\right)$ and the constants $\alpha_{3}$ and $\alpha_{4}$. Note from Eqs. (12), (13), (18), (19), (20), and (24) that the heat flux at the surface of the sensor depends linearly on the parameters $\alpha_{3}, \alpha_{4}$, and $g_{0}$. Furthermore, function (25) depends nonlinearly on the parameters $\alpha_{1}, \alpha_{r 2}$, and $\rho_{1} c_{p 1} /\left(\rho_{2} c_{p 2}\right)$. This type of dependence permits to use a linear parameter estimation solver to obtain the parameters $\alpha_{3}$, $\alpha_{4}$, and $g_{0}$. A nonlinear minimization algorithm can be used to obtain the constants $\alpha_{1}, \alpha_{r 2}$, and $\rho_{1} c_{p 1} /\left(\rho_{2} c_{p 2}\right)$.

\section{NUMERICAL IMPLEMENTATION}

Equation (24) was implemented in FORTRAN. An algorithm developed in [1] was used to determine the eigenvalues from the transcendental equation (20). Initially, a FORTRAN program was written to implement Eq. (9). Later on, this computer program was modified to implement Eq. (24). Extensive numerical testing was carried out to make sure that the results from both programs agree to sixteen decimal places.

\section{THERMAL EQUILIBRIUM PREDICTION ALGORITHM}

Thermal equilibrium predictions are carried out using a parameter estimation hybrid algorithm [2] and the numerical implementation of Eq. (24). For the sake of describing the key aspects of this hybrid algorithm, Eq. (24) is written in a more convenient form as

$$
-\left.k_{r 2} \frac{\partial T_{2}}{\partial r}\right|_{r=b}=\sum_{j=1}^{3} C_{j} \Psi_{j}(t)
$$

where $C_{1}=g_{0}, C_{2}=\alpha_{3}, C_{3}=\alpha_{4}$, and

$$
\begin{gathered}
\Psi_{1}(t)=-\alpha_{r 2} d \sum_{m=1}^{\infty} \frac{a J_{1}\left(\eta_{m 0} a\right)}{\eta_{m 0} \lambda_{m 0}^{2} N_{m 0}^{*}} \\
\times\left.\frac{\partial F_{2, m 0}}{\partial r}\right|_{r=b}\left[1-\exp \left(-\lambda_{m 0}^{2} t\right)\right] \\
\Psi_{2}(t)=-\alpha_{r 2} \sum_{m=1}^{\infty} \frac{\exp \left(-\lambda_{m 0}^{2} t\right)}{N_{m 0}^{*}} \frac{a d J_{1}\left(\eta_{m 0} a\right)}{\eta_{m 0}} \\
\times\left.\frac{\partial F_{2, m 0}}{\partial r}\right|_{r=b}
\end{gathered}
$$




$$
\begin{aligned}
\Psi_{3}(t) & =-\left.\alpha_{r 2} \sum_{m=1}^{\infty} \frac{d \exp \left(-\lambda_{m 0}^{2} t\right)}{\gamma_{m 0} N_{m 0}^{*}} \frac{\partial F_{2, m 0}}{\partial r}\right|_{r=b} \\
\times & \left\{C_{m 0}\left[b J_{1}\left(\gamma_{m 0} b\right)-a J_{1}\left(\gamma_{m 0} a\right)\right]\right. \\
+ & \left.D_{m 0}\left[b Y_{1}\left(\gamma_{m 0} b\right)-a Y_{1}\left(\gamma_{m 0} a\right)\right]\right\}
\end{aligned}
$$

Let $\Theta$ represent the heat flux sensor signals at discrete intervals of time, the functional to be minimized is defined as

$$
S=\sum_{p=1}^{M}\left[\Theta\left(t_{p}\right)-\sum_{j=1}^{3} C_{j} \Psi_{j}\left(t_{p}\right)\right]^{2}
$$

where $\left\{t_{1}, t_{2}, \ldots, t_{M}\right\}$ are the times at which the voltage measurements are taken and $M$ is the number of measurements considered in the minimization. The method selected to minimize Eq. (30) is a technique without derivatives modified by Powell (in Brent [4]). Powell's method is used to minimize Eq. (30) with respect to the parameters $\alpha_{1}, \alpha_{r 2}$, and $\rho_{1} c_{p 1} /\left(\rho_{2} c_{p 2}\right)$. For each set of parameters selected by Powell's algorithm [3, 4], a least-squares procedure is applied to solve the linear system

$$
\begin{gathered}
\frac{\partial}{\partial C_{j}}\left\{\sum_{p=1}^{M}\left[\Theta\left(t_{p}\right)-\sum_{j=1}^{3} C_{j} \Psi_{j}\left(t_{p}\right)\right]^{2}\right\}=0 \\
\text { for } j=1,2,3
\end{gathered}
$$

The estimation procedure begins by providing initial guesses for the parameters $\alpha_{1}, \alpha_{r 2}$, and $\rho_{1} c_{p 1} /\left(\rho_{2} c_{p 2}\right)$. Then the solution of the linear system described by Eq. (31), yields the coefficients $\left\{C_{1}, C_{2}, C_{3}\right\}$. Once the coefficients $C_{j}$ are known, the value of the functional $S$ is calculated and returned to Powell's algorithm. At this stage, Powell's algorithm provides a second estimation for the parameters $\alpha_{1}, \alpha_{r 2}$, and $\rho_{1} c_{p 1} /\left(\rho_{2} c_{p 2}\right)$. This process is repeated until a predefined convergence criterion for the functional $S$ is satisfied. Figure 7 shows a simplified flowchart of the hybrid algorithm.

The major goal in this study is to reduce the assay time in calorimeter measurements. Once the parameter estimation problem described above is solved, the estimated parameters are substituted back into Eq. (24) and a thermal equilibrium prediction is obtained by taking the limit of Eq. (24) as $t \rightarrow \infty$. Note that only the first summation term in Eq. (24) survives in the limit as $t \rightarrow \infty$. This is to be expected since this term contains the volumetric heat generation constant $g_{0}$ which is a measure of the heat produced by the radioactive decay of the material being assayed in the calorimeter. The value of $g_{0}$ obtained from the parameter estimation problem described above and the steady-state closed form solution can also be used to predict thermal equilibrium. Equation (10) is a one-dimensional solution of the heat conduction equations in the calorimeter. These equations have the form

$$
\begin{gathered}
k_{1} \frac{1}{r} \frac{\partial}{\partial r}\left(r \frac{\partial T_{1}}{\partial r}\right)+g_{0}=\rho_{1} c_{p 1} \frac{\partial T_{1}}{\partial t} \\
k_{r 2} \frac{1}{r} \frac{\partial}{\partial r}\left(r \frac{\partial T_{2}}{\partial r}\right)=\rho_{2} c_{p 2} \frac{\partial T_{2}}{\partial t}
\end{gathered}
$$

As time tends to infinity, $\partial T_{1} / \partial t$ and $\partial T_{2} / \partial t$ in Eqs. (32) and (33) tend to zero to produce

$$
\begin{gathered}
k_{1} \frac{1}{r} \frac{d}{d r}\left(r \frac{d T_{1}}{d r}\right)+g_{0}=0 \\
\frac{1}{r} \frac{d}{d r}\left(r \frac{d T_{2}}{d r}\right)=0
\end{gathered}
$$

Equations (34) and (35) are the steady-state versions of Eqs. (32) and (33). They can be solved using the continuity of temperature and heat flux at the interface between the sensor and the inner enclosure of the calorimeter. These conditions can be stated as

$$
\begin{gathered}
T_{1}(a)=T_{2}(a) \\
k_{1} \frac{d T_{1}}{d r}=k_{r 2} \frac{d T_{2}}{d r} \text { at } r=a
\end{gathered}
$$

The boundary condition at $r=b$ used to obtain Eq. (10) is also used to solve Eqs. (34), (35), (36), and (37). This condition reads

$$
T_{2}(b)=T_{s}
$$

where $T_{s}$ is the temperature of the outer surface of the tube that surrounds the sensor. The solutions of Eqs. (34) and (35) are

$$
\begin{aligned}
& T_{1}(r)=A-\frac{g_{0} r^{2}}{4 k_{1}} \\
& T_{2}(r)=C+D \ln r
\end{aligned}
$$

The constants $A, C$, and $D$ can be obtained applying conditions (36)-(38). After calculating these constants, Eq. (40) takes the form

$$
T_{2}(r)=T_{s}-\frac{g_{0} a^{2}}{2 k_{r 2}} \ln \left(\frac{r}{b}\right)
$$




$$
-\left.k_{r 2} \frac{\partial T_{2}}{\partial r}\right|_{r=b}=\frac{g_{0} a^{2}}{2 b}
$$

After calculating $g_{0}$ using the thermal equilibrium prediction algorithm, Eq. (42) can also be used to obtain the steady-state heat flux.

\section{STRUCTURE OF THE THERMAL EQUILIBRIUM PREDICTION ALGORITHM AND NUMERICAL PROCESSING OF THE HEAT FLUX SENSOR SIGNAL}

The objective of thermal equilibrium prediction is to reduce the total assay time by a significant amount and still keep the prediction percentage error at an acceptable level. The data sets that contain the thermal response of the heat flux sensor are modified first to define the initial time and voltage, and the thermal equilibrium prediction region. A fraction of the total assay time is defined first prior to the definition of this region. Once the fraction of the total assay time is calculated, the definition of the thermal equilibrium region is made based on the shape of the heat flux sensor signal at the earliest stages of the measurement. If the sensor signal contains the effects of the opening and closing of the calorimeter, see Figs. 2 and 4, this part of the signal is discarded. Otherwise, the definition of the thermal equilibrium prediction region is done as shown in Figs. 1 and 3. Figures $2-4$ show the time, $t_{i}$, and voltage, $V_{i}$, used to transform the time and voltage scales so that the thermal equilibrium region starts at time equal to zero and at voltage equal to zero.

The calorimeter heat flux sensor voltage readings are taken with a digital multi-meter and they contain some degree of noise. The voltage readings are taken at time intervals, $\Delta t_{a}$, defined by the operator of the data acquisition software. The data acquisition sampling interval, $\Delta t_{a}$, is not the optimum discretization interval, $t_{p}-t_{p-1}$, that should be used to define the number of measurements used for the minimization of Eq. (30). For this reason, numerical experiments were carried out using 18 data sets to estimate the optimum size of the discretization interval that should be used in Eq. (30). It was found empirically that two numerical interpolations applied in series provided reasonable results. Initially, a prediction region that represents a fraction of the total assay time is defined. Next, two numerical interpolations are applied to this prediction region. First, the experimental data is interpolated linearly using a time step, $\Delta t$, equal to 16 seconds. Second, a cubic or linear interpolation that uses Lagrange's classical formula and Neville's algorithm [3] is applied to divide the prediction region into 5 to 3 data points. These data points represent the number of measurements, $M$, considered in the minimization of Eq. (30). Figure 5 shows a sketch of the numerical processing of the experimental data contained in the thermal equilibrium prediction region. The data points generated in the prediction region are fed to Powell's algorithm and the hybrid algorithm starts as explained in the paragraph subsequent to the statement of Eq. (30). A flow chart of the hybrid algorithm is shown in Fig. 7.

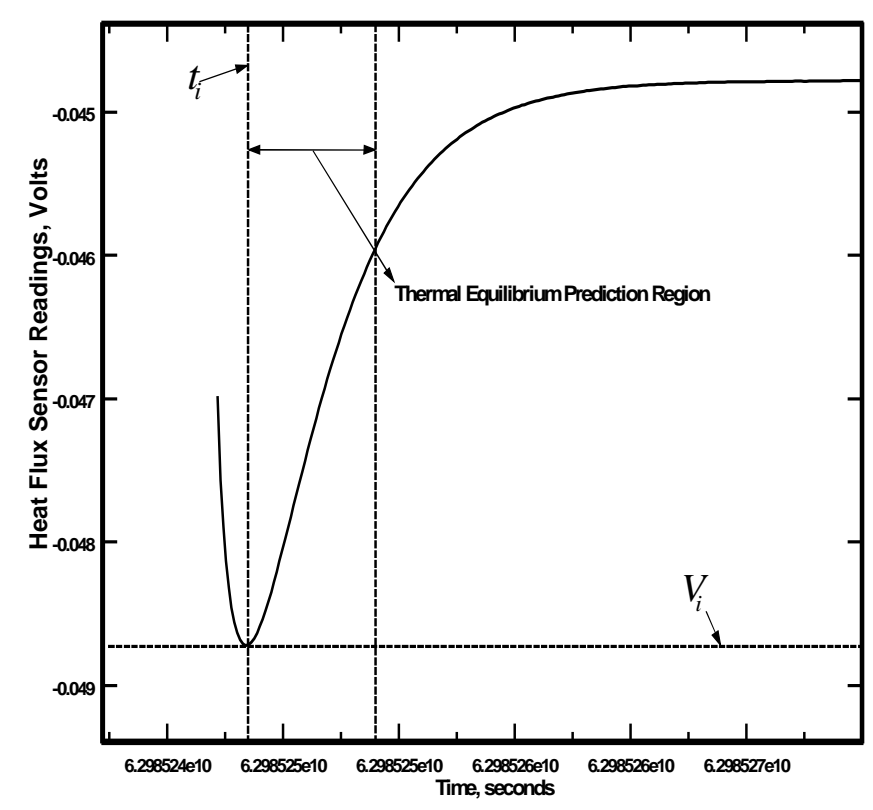

Figure 2. Typical heat flux sensor calorimeter response that includes the effects of opening and closing the calorimeter cover to insert the sample. The location of the minimum at $\left(t_{i}, V_{i}\right)$ is also depicted.

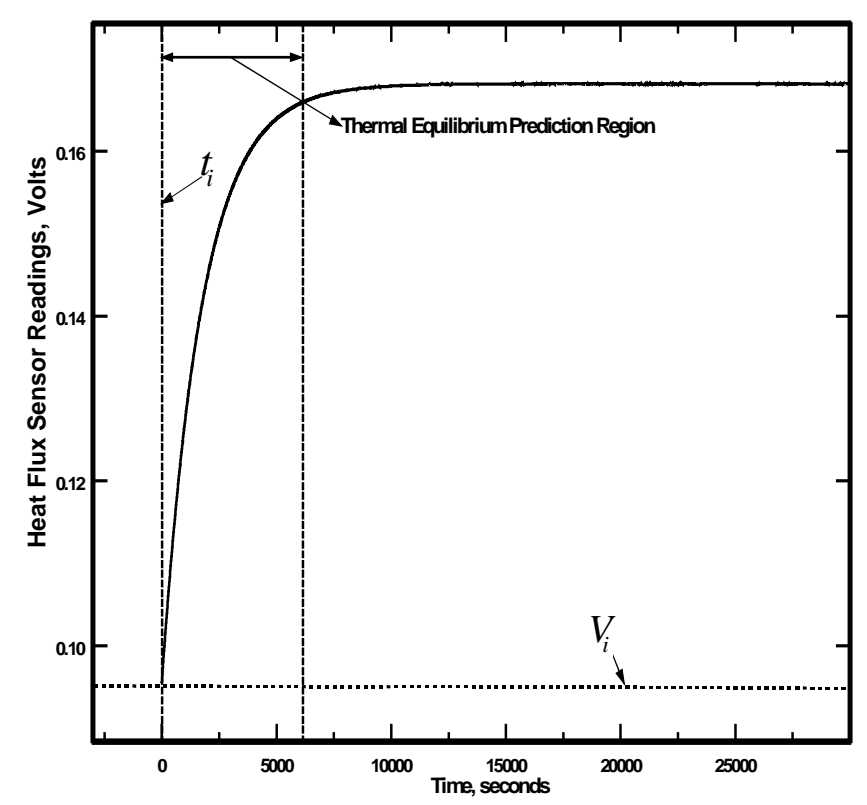

Figure 3. Typical heat flux sensor calorimeter response depicting the thermal equilibrium prediction region. 


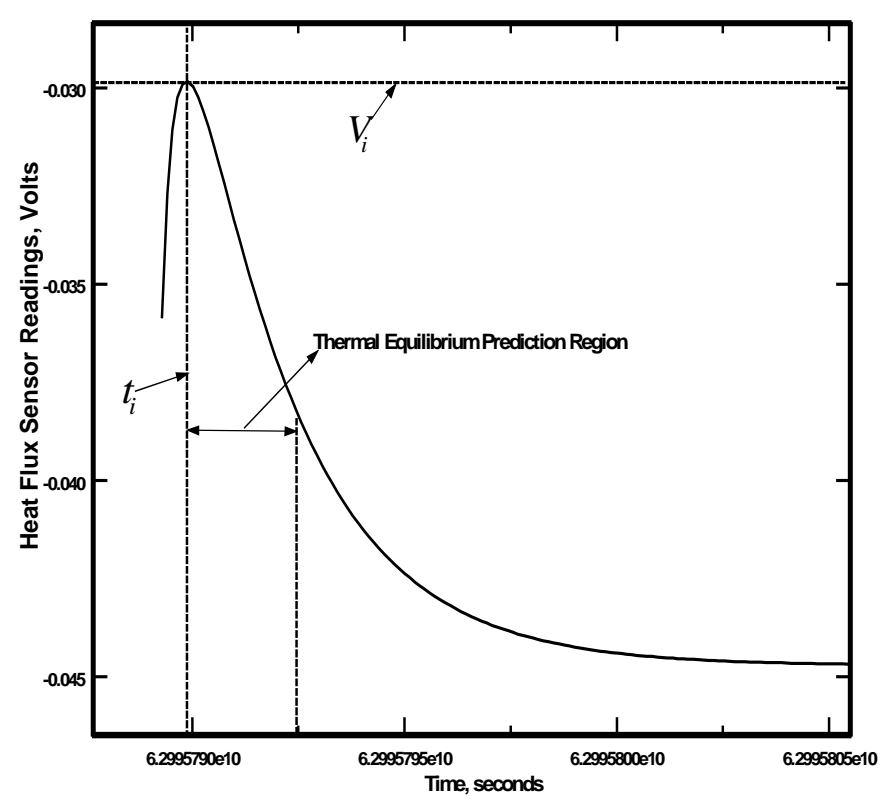

Figure 4. Typical heat flux sensor calorimeter response that includes the effects of opening and closing the calorimeter cover to insert the sample. The location of the minimum at $\left(t_{i}, V_{i}\right)$ is also depicted.

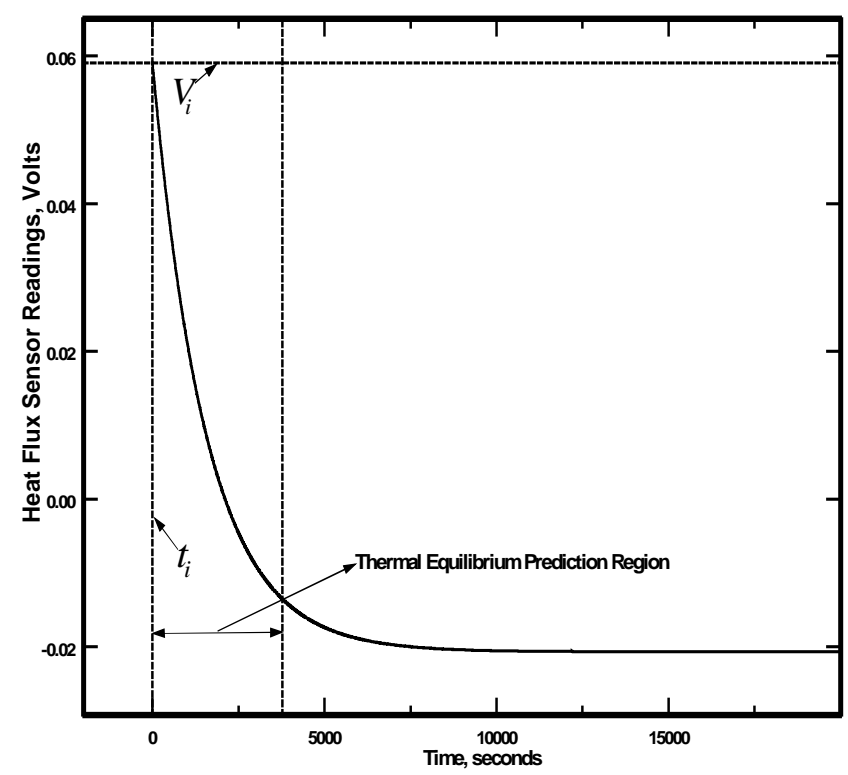

Figure 5. Typical heat flux sensor calorimeter response depicting the thermal equilibrium prediction region.

\section{THERMAL EQUILIBRIUM PREDICTION RESULTS}

Thermal equilibrium predictions were carried out using 330 data sets from different calorimeters. One hundred and sixty four predictions using 164 data sets from the highly enriched uranium (HEU) calorimeter have an average percentage error of $0.085 \%$. This average is calculated using an error defined as

$$
e=\left|\frac{E-P}{E}\right| \times 100 \text {. }
$$

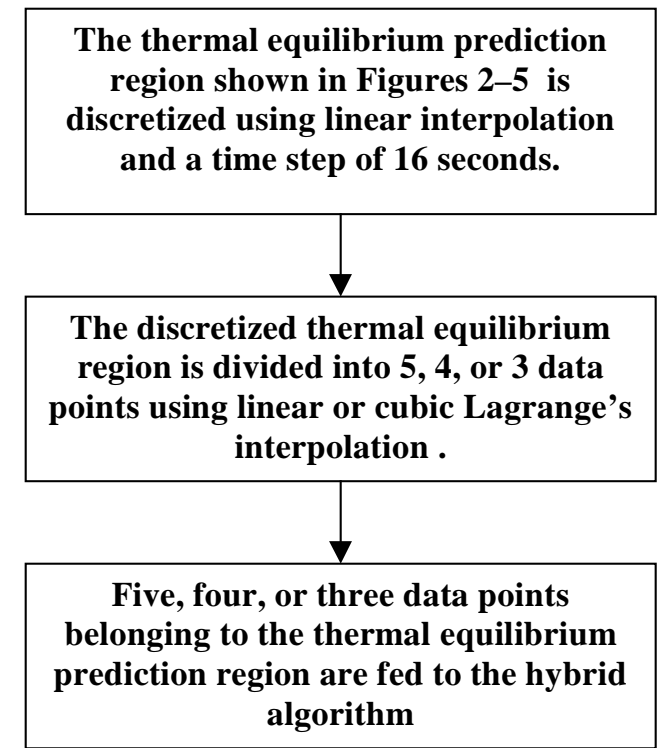

Figure 6. Numerical processing applied to the data set represented by the thermal equilibrium prediction region.

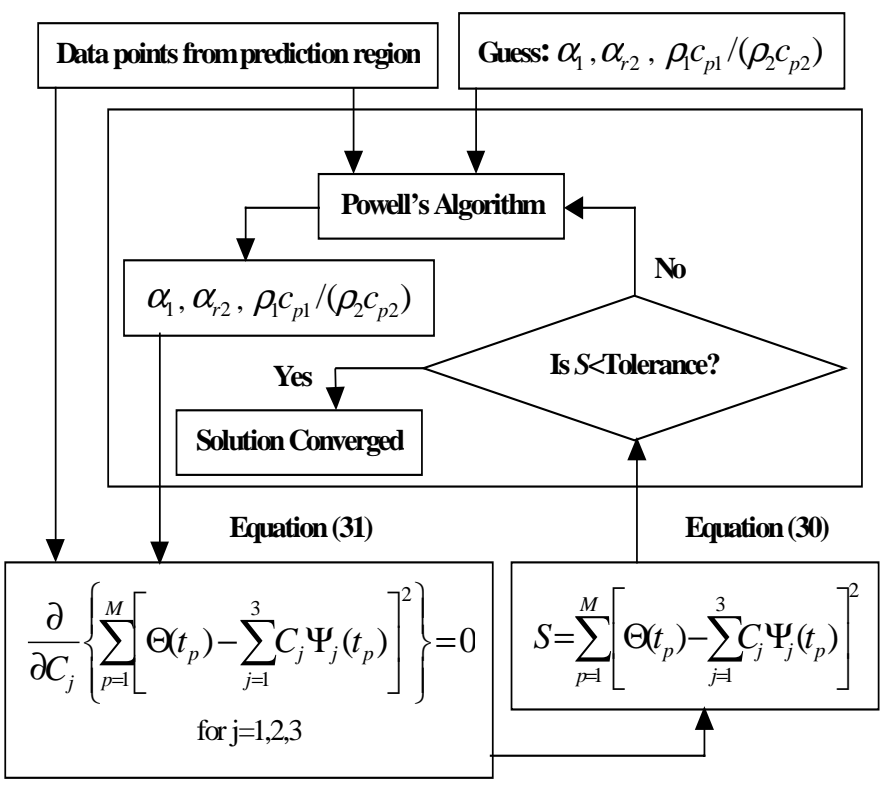

Figure 7. Flow chart of the hybrid algorithm that estimates the parameters used to carry out the thermal equilibrium prediction using Eq. (24). 


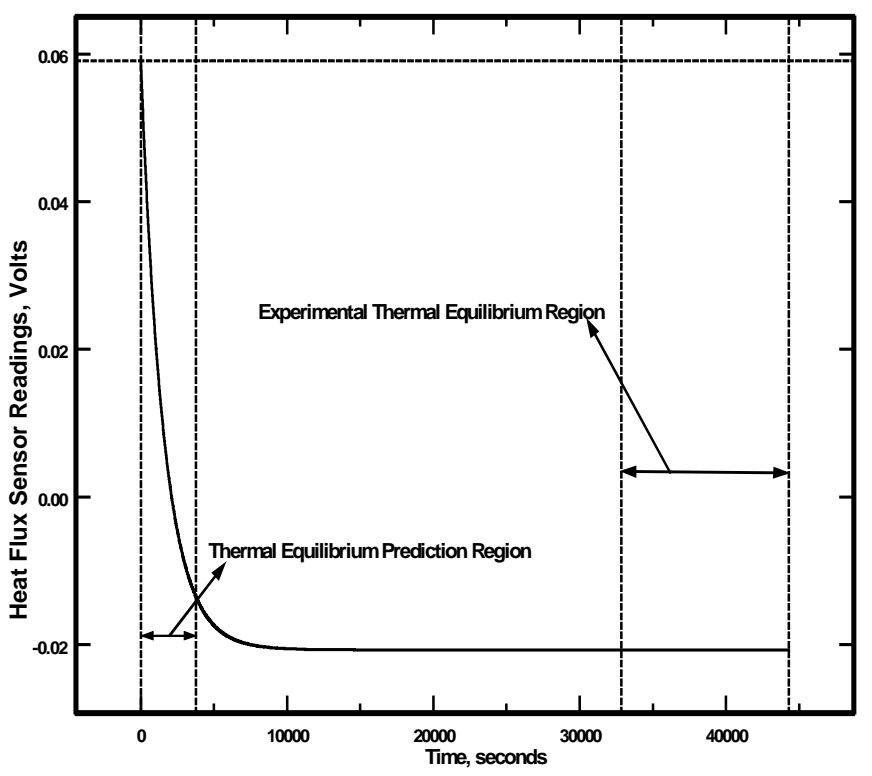

Figure 8. Typical calorimeter voltage readings showing the experimental and prediction thermal equilibrium regions.

Table 1. Thermal equilibrium predictions average error calculated using the error defined by Eq. (43) and data sets for each calorimeter.

\begin{tabular}{|l|c|c|c|}
\hline Calorimeter & $\begin{array}{c}\text { Average } \\
\text { Error }\end{array}$ & $\begin{array}{c}\text { Time } \\
\text { Ratio }\end{array}$ & $\begin{array}{c}\text { Mean }(E-P), \\
\text { Volts }\end{array}$ \\
\hline HEU & $0.085 \%$ & 0.208 & $2.723 \mathrm{E}-6 \pm 1.70 \mathrm{E}-5$ \\
\hline RFETS & $0.145 \%$ & 0.202 & $9.109 \mathrm{E}-6 \pm 1.63 \mathrm{E}-5$ \\
\hline SS & $0.179 \%$ & 0.205 & $4.507 \mathrm{E}-7 \pm 1.46 \mathrm{E}-6$ \\
\hline
\end{tabular}

The symbol $E$ appearing in Eq. (43) is the experimental thermal equilibrium obtained taking the arithmetic average of the voltage data in the experimental thermal equilibrium region, and $P$ is the predicted thermal equilibrium obtained using the model Eq. (24). A typical experimental thermal equilibrium region is depicted in Fig. 8. One hundred and seventeen thermal equilibrium predictions using 117 data sets from the Rocky Flats Environmental Technology site (RFETS) have an average percentage error of $0.145 \%$. Results for the solid state calorimeter (SS) show that 69 thermal equilibrium predictions have an average error of $0.179 \%$. Table 1 summarizes these results. Furthermore, the second column of Table 1 shows the average percentage error calculated using the error defined by Eq. (43), the third column shows the ratio of the time used to carry out the prediction to the total assay time, the fourth column shows the average of the difference, $|E-P|$, in Volts. The fourth column in Table 1 can be converted into Watts dividing by the sensitivity of each calorimeter given in Volt/Watt. For the HEU calorimeter, this average produces: $\operatorname{Mean}(P-E)=9.55 \mathrm{E}-5 \pm 5.95 \mathrm{E}-4$ Watts. The RFETS row yields: $\operatorname{Mean}(P-E)=6.66 \mathrm{E}-4 \pm 1.19 \mathrm{E}-3$ Watts. For the SS calorimeter: $\operatorname{Mean}(P-E)=4.29 \mathrm{E}-5 \pm 1.39 \mathrm{E}-4$ Watts. Table 1 shows that the average percentage error for the 330 equilibrium predictions made in this study is only $0.136 \%$.

\section{REMARKS AND CONCLUSIONS}

A thermal equilibrium prediction algorithm is developed using an exact heat conduction model. The results shown in Table 1 show that this model can reduce the total assay time significantly and still maintain a reasonable error level. Furthermore, this thermal equilibrium prediction algorithm can reduce the assay time in large volume calorimeters. Large volume calorimeters have longer assay times.

The percentage of the total assay time used in this study, $20 \%$, can be reduced further by developing a criterion to estimate the optimal final prediction time. This criterion may reduce the percentage of the total assay time somewhere between $10 \%$ and $20 \%$. One approach that can be used to develop this criterion is to look at the behavior of the first and second derivatives of the calorimeter response at the early stages of the measurement. Furthermore, this criterion can be used to develop a real time algorithm that will decide when to stop acquiring data and carry out the thermal equilibrium prediction.

Typical calorimeter calibration curves are constructed using the arithmetic average of the voltages at thermal equilibrium, see Fig. 8. A calibration curve can also be constructed using the model Eq. (24) by carrying out thermal equilibrium predictions considering the total assay time. A calibration curve constructed with the model Eq. (24) would provide more consistency when predictions using a fraction of the total assay time are carried out utilizing Eq. (24).

\section{ACKNOWLEDGMENTS}

The author wishes to acknowledge the support of the U. S. Department of Energy, Office of Safeguards and Security. Also, the author would like to acknowledge the valuable comments and encouragement of Cliff Rudy.

\section{REFERENCES}

[1] Aviles-Ramos, C., "Exact Solution of Heat Conduction in a Two-Domain Composite Cylinder with an Orthotropic Outer Layer", Proceedings of the $35^{\text {th }}$ National Heat Transfer Conference, Anaheim, California, 2001.

[2] Aviles-Ramos, C., "Estimation of Thermophysical Properties of an Orthotropic Region of a Two-Layer Body Using the Exact Solution," Ph.D. Dissertation, University of Texas at Arlington, Arlington, Texas, 2000.

[3] Press, W. H., Teukolsky, S. A., Vetterling, W. T., and Flannery, B. P., Numerical Recipes in FORTRAN: The Art of Scientific Computing, Cambridge University Press, London, UK, 1992.

[4] Brent, R. P., Alogrithms for Minimization Without Derivatives, Prentice Hall, Inc. Englewood Cliffs, New Jersey, 1973. 